\title{
EXPLORATION OF PLANT SPECIES USED BY THE TRIBAL, KOTAS FOR THEIR MEDICINAL USES IN THE UPPER REACHES OF KOTAGIRI, THE NILGIRIS, WESTERN GHATS
}

\author{
Ganesn C.M. ${ }^{*}$, K.Manikandan ${ }^{2}$ and S.Paulsamy ${ }^{2}$ \\ ${ }^{1}$ Department of Botany, Government Arts College, Udumalpet. \\ ${ }^{2}$ Department of Botany, Kongunadu Arts and Science College, Coimbatore. \\ *E.mail: bioganesan@gmail.com
}

\begin{abstract}
The medicinal plants provide an efficient local aid to health care and disease free life. The present investigation has been under taken in Trichigady, Kotagiri terrace, Nilgiri biosphere during the study period June 2016 to December 2016. In order to study that the traditional uses of these folk medicinal practice day todays life it as resulted providing information of 66 wild plants. Out of the 140 species are comprised in the families like Acanthaceae, Amaranthaceae, Convolvalaceae, Euphorbiaceae, Rutaceae, Solanaceae etc. In this case study, they are used 66 plants in medicinal purpose and 74 plants are used as edible food respectively. So this investigation is held us to understand how indigenous knowledge possess by Kota tribal of study area.
\end{abstract}

Keywords: Tribal, Kotas, Western Ghats, Kotagiri.

\section{INTRODUCTION}

The medicinal plants represent not only a valuable part of India's biodiversity but also a source of great knowledge. The WHO has listed 21,000 plants that are used as medicine around the world. India has a rich medicinal plant flora of some 25,000 species (Bhattacharjee, 2001). There are 1532 edible wild food species in India, mostly from Western Ghats and Himalayan region (Kujur, 1989). Only about 2 percent of more than 250,000 species of higher plants have been carefully evaluated for medicinal activity (Deepak Chopra and David Simson, 2000).

The Trichigady village is a part of Kotagiri, Nilgiri Hills,the Western Ghats, Tamil Nadu, India were selected for the present study to obtain the medicinal and edible plants information from Kota tribal village. The study are located at latitudes $10^{\circ}$ $45^{\prime}$ to $12^{\circ} 15^{\prime} \mathrm{N}$ and longitudes $76^{\prime}$ to $77^{\prime} 15^{\circ} \mathrm{E}$. The elevation of the study area is $2320 \mathrm{~m}$ above MSL. The species richness is high in general and many of the species showed variations in their populations which aided the species for better distribution, survival and perpetuation in different microclimatic conditions. In addition to commonly distributed species, many red listed species with various economic uses are also distributed in the existing vegetations (Prasad and Balasubramaniam, 1996; Murugesan, 2005). Further, due to illegal exploitation, it has been determined that many species attained low status of population size (Paulsamy et al., 2008).
Principally, earlier studies in the Nilgiri Biosphere Reserve have dealt with medicinal species and little attention was paid to wild edible plants (Perumal Samy and Ignacimuthu 2000; Rajan et al., 2003; Rajasekaran et al., 2005; Udayan et al., 2007; Revathi and Parimelazhagan 2010; Poongodi et al., 2011). Vedavathy (2002) gave an account of the real alternatives of the tribal medicine.

Rajan, et al. (2002) discussed the flowering plants uses for remedial purposes by the Kattunayakas of Nilgiris, Tamilnadu. Rajendran, et al. (2004) gave a detailed account on medicinal plants and their utilization by villages in Southwestern Ghats, Tamilnadu. Ganesan, et al. (2004) reported the ethno medicinal aspects of 45 species of plants used by the Paliyan and Pulayan tribe of lower Palani hills Tamilnadu. Hema, et al. (2006) identified a total of 15 taxa and recognized as being used by Kurumba and Paniya of Wyanad district, Kerala.

In recent times, due to inexplicable reasons there has been a rapid decline and deterioration of folk medical practice/traditional knowledge. Therefore, the revival and revitalization of this folk medical system is to be improved on its scientific base. In such cases, the tribal medicine can provide safe, stable, standardized and therapeutically effective drugs not only to the tribal communities but also to the other populations can affordable cost. 


\section{MATERIALS AND METHODS}

Each and every ethnobotanical work has various activities. They are field trip, observations, identification retrieving the medicinal properties and mode of preparation of drug from the plants by Kota tribal.

Trichigudy village is located in a part of Kotagiri, Nilgiri Hills (NBR), the Western Ghats, Tamilnadu. The present investigation was undertaken with a view of studying the uses of plants in study area. Intensive botanical exploration trip were undertaken in and around trichigady during the period of November 2013 - April 2014. In this period per a month 4 trips were done. During this trip we have collected the information about the edible plants and the medicinal plants including their parts uses and aliments were obtained from Kota tribal.

The voucher specimen plants collected were identified with the help of Flora of Presidency of Madras by J.S. Gamble (1936) and Flora of
Tamilnadu and Carnatic by K.M. Mathew (1983).

The medicinal plants collected in this way are tabulated. They are documented, both family and genus are arranged according to the alphabetical order. The botanical names followed by author citation and synonyms of the plant species, local name of the plant species also provided. Most of the plants are used as a medicine rest of them served as an edible plants.

\section{RESULTS AND DISCUSSION}

The study area Trichigady Kota village is located in a part of Kotagiri, Nilgiri Hills [NBR], the Western Ghats, Tamilnadu. About 140 species are collected spreading over - families (Table 1 - 3). They are identified for their medicinal and edible importance with the help of Kota tribal people. The ethnobotanical studies of some tribal area are reported (Rajan and Sethuraman, 1991; Rajesekaran etal., 2005; Yasodharan and Sujana, 2007; Rasingam and Rehel, 2009; Rasingam, 2010; The taxonomic position were identified through relevant literatures.

Table 1. List of medicinal plants used by Kota tribal in study area.

\begin{tabular}{|c|c|c|c|c|}
\hline S.No & Scientific name & Family & Parts used & Ailments cured \\
\hline 1 & Acacia leucophloea(Roxb.) Willd. & Mimosaceae & Bark & $\begin{array}{l}\text { Bone Fracture, Cuts and } \\
\text { burns }\end{array}$ \\
\hline 2 & AcalyphafruticosaForsskal & Euphorbiaceae & $\begin{array}{l}\text { Whole } \\
\text { Plant }\end{array}$ & To Control worms \\
\hline 3 & AchyranthusbidentataBlumeic & Amaranthaceae & Leaf & To cure skin disorders \\
\hline 4 & Aeglemarmelos(L.) Corr. Serr. & Rutaceae & Bark & Diarrhea \\
\hline 5 & Alangiumsalvifoliumvar.hexapettalumWang & Alangiaceae & Bark & Snake antidote\& Paralysis \\
\hline 6 & Albiziaamara(Roxb.) & Mimosaceae & $\begin{array}{l}\text { Leaves } \\
\text {,bark }\end{array}$ & $\begin{array}{l}\text { For hair growth, Cuts and } \\
\text { burns }\end{array}$ \\
\hline 7 & Aloe vera(L).Burm.f. & Xanthorrhoeaceae & Fruit & Skin disorder \\
\hline 8 & AndrographispaniculataVahl & Acanthaceae & Roots & Snake antidote \\
\hline 9 & AndrographisserphilifoliaVahl. & Acanthaceae & Roots & Snake antidote \\
\hline 10 & AnogeissuslatifoliaWallich ex Guill. \&Perr. & Combretaceae & Bark & Stomach ache \\
\hline 11 & Ageratum conyzoidesLinn. & Asteraceae & Leaf & Wound healer \\
\hline 12 & ArgyreiaspeciosaBurm.f & Convolvulaceae & Roots & Fever and headache \\
\hline 13 & ArisaemaleschenaultiiBlume & Araceae & Roots & Snake antidote \\
\hline 14 & AzadirachtaindicaAdr.Juss. & Meliaceae & Bark & $\begin{array}{l}\text { Leaves To Control worms, } \\
\text { Mouth Ulcer }\end{array}$ \\
\hline 15 & BarleriabuxifoliaL. & Acantaceae & Roots & Stomach pain \\
\hline 16 & Bauhinia racemosa $\mathrm{L}$. & Caesalpiniaceae & Bark & Dysentery \\
\hline 17 & BerberiestinctoriaLesch. & Berberidaceae & Leaves & $\begin{array}{l}\text { Polycystic ovarian } \\
\text { syndrome }\end{array}$ \\
\hline 18 & Calotropisgigantea(L) R.Br & Asclepiadaceae & Leaves & Foot problems \\
\hline 19 & Canthiumcoromandelicum(Burm.f.) Alston & Rubiaceae & Bark & Fever \\
\hline 20 & CapparissepiariaL. & Capparaceae & Leaves & Hip pains, dysentery \\
\hline 21 & CappariszeylanicaL. & Capparaceae & Leaves & Breathing problems \\
\hline 22 & Cassia occidentalisL. & Fabaceae & Roots & Swellings over body \\
\hline 23 & Celtisphilippensiswight & Ulmaceae & Bark & Digestion problems \\
\hline 24 & CentellaasiaticaL. & Mackinlayaceae & Leaves & Syphilis \\
\hline 25 & ChloroxylonswieteniaRoxb. & Rutaceae & Inner bark & Tooth problems \\
\hline 26 & Chenopodium album Linn. & Chenopodiaceae & Leaves & Kidney stones \\
\hline
\end{tabular}


CissampelospareiraL.

27 Cocciniagrandis(L)J.voigt

28 Cocciniaindica

29 Colocassiaesculenta(Linn.) Schott. \&Endl.

30 CordiamonoicaRoxb.

31 Curcuma pseudomontanaJ.Graham.

32 Curcuma neilgherrensiswight

33 CyanodondactylonL.

34 DaturametelL.

35 DichrostachysscinereaWight et al.

36 Dodonaea viscose (Linn.) Jacq.

$37 \quad$ Elaeagnuskologaschlecht.

38 ErythroxylonmonogynumRoxb.

39 Gaultheria fragrantissimaWall.

40 GivotiamollucanaL.

41 GmelinaarboreaRoxb.

42 Hibiscus micranthusL.f.

43 Ipomeaobscura(L.) Ker Gawl.

44 JasminumauriculatumVahl.

45 JatrophacurcasL

46 Leucasaspera(Willd.) Link

47 Manilkarahexandra(Roxb.)Dubard.

$48 \quad$ Moringaconcanensis

49 Narinjicrenulata(Roxb.) Nicols.

$50 \quad$ Nerium oleander L.

51 PartheniumhysterophorusL.

52 Phyllanthusdebiliskleinex wild

53 PleiospermumalatumWight\&Arn.

54 Prunella vulgaris Linn.

55 RaphidophorapertusaHassk

56 RicinuscommunisL.

57 SapindusemarginataVahl.

58 SiegesbeckiaorientalisLinn.

59 SolanumnigrumL.

60 SolanumsurattenseBurm.F.

61 Tephrosiapurpurea(Linn.)Pers.

62 TerminaliachebulaRetz.

63 Wattakakavolubilis(Linn.F)stapf.

64 Xanthium indicum J. Koenig ex Roxb.

65 ZiziphusmauritianaLamk

66 ZiziphusrugosaLamk

$\begin{array}{ccl}\text { Menispermaceae } & \text { Leaves } & \text { Snake antidote } \\ \text { Cucurbitaceae } & \text { Leaves, } & \text { Throat pain } \\ & \text { tubers } & \\ \text { Cucurbitaceae } & \text { Roots } & \text { Antidote } \\ \text { Araceae } & \text { Leaves } & \text { Stomach disorder } \\ \text { Boragianaceae } & \text { Leaves } & \text { Chest pains } \\ \text { Zingiberaceae } & \text { Gingers } & \text { Wounds and cuts } \\ \text { Zingiberaceae } & \text { Rhizome } & \text { Anti diabetic } \\ \text { Poaceae } & \text { Fibre } & \text { Headache } \\ \text { Solanaceae } & \text { Leaves } & \text { Pain relief } \\ \text { Mimosaseae } & \text { Fibre paste } & \text { Vomiting } \\ \text { Sapindaceae } & \text { Leaves } & \text { Joint sprains, Fractures } \\ \text { Elaeagnaceae } & \text { Leaves } & \text { Antifeedants } \\ \text { Erythroxylaceae } & \text { Bark } & \text { Skin disorder } \\ \text { Ericaceae } & \text { Leaves } & \text { Antiseptic } \\ \text { Euphorbiaceae } & \text { Bark } & \text { Breathing problems } \\ \text { Verbenaceae } & \text { Leaves } & \text { Stomach ache } \\ \text { Malvaceae } & \text { Roots } & \text { Swellings over body } \\ \text { Convolvulaceae } & \text { Leaves } & \text { Sprain, stomach ache } \\ \text { Acanthaceae } & \text { Stems\& } & \text { Bone fractures } \\ & \text { roots } & \\ \text { Euphorbiaceae } & \text { Inner bark } & \text { Cold and fever } \\ \text { Lamiaceae } & \text { Whole } & \text { Typhoid } \\ & \text { Plant } & \\ \text { Sapotaceae } & \text { Bark } & \text { Hip pains } \\ \text { Moringaceae } & \text { Bark \& } & \text { De-worming, Dysentery \& } \\ & \text { leaves } & \text { fever } \\ \text { Rutaceae } & \text { Leaves } & \text { Leg pains } \\ \text { Apocyanaceae } & \text { Leaves } & \text { For speech to children } \\ \text { Asteraceae } & \text { Leaves } & \text { Cuts and burns } \\ \text { Euphorbiaceae } & \text { Leaves } & \text { Jaundice } \\ \text { Rutaceae } & \text { Bark } & \text { Chest pains } \\ \text { Lamiaceae } & \text { Roots } & \text { Hematinic } \\ \text { Araceae } & \text { Whole } & \text { Swellings in groin joints } \\ & \text { Plant } & \\ \text { Euphorbiaceae } & \text { Seed oil } & \text { Dysentery } \\ \text { Sapindaceae } & \text { Inner bark } & \text { Tooth problems } \\ \text { Asteraceae } & \text { Leaves } & \text { Insect bites \& rashes } \\ \text { Solanaceae } & \text { Whole } & \text { Stomach ache\& fever } \\ & \text { Plant } & \\ \text { Solanaceae } & \text { Fruits, } & \text { Vomiting \&Tooth paste } \\ & \text { leaves } & \\ \text { Fabaceae } & \text { Leaves } & \text { Antitumor } \\ \text { Combretaceae } & \text { Fruits } & \text { Cough and Fever } \\ \text { Asclepiadaceae } & \text { Leaves } & \text { Dysentery } \\ \text { Asteraceae } & \text { Leaves } & \text { Dog bite } \\ \text { Rhamnaceae } & \text { Bark } & \text { Dysentery } \\ \text { Rhamnaceae } & \text { Bark } & \text { Dysentery } \\ & & \\ & & \\ & & \end{array}$

Table 2. List of edible plants used by Kota tribal in study area.

\begin{tabular}{clccc}
\hline S.No & \multicolumn{1}{c}{ ScientificName } & Family & Tamil Name & Edible Part \\
\hline 1 & Acacia pennata(L.)Willd. & Mimosaceae & Seengai & Leaf \\
2 & Alternantherasessilis(L.) R. Br. ex DC. & Amaranthaceae & Ponnakanni & Leaf \\
3 & AmaranthuscaudatusL. & Amaranthaceae & keeraiThandu & Leaf \\
4 & AmaranthusgraecizansL. & Amaranthaceae & Sirukeerai & Leaf \\
5 & AmaranthusspinosusL. & Amaranthaceae & Mullu & Leaf \\
6 & AmaranthusviridisL. & Amaranthaceae & Pattikerae & Leaf
\end{tabular}


Asparagus racemosusWilld.

Nastusborbonicus].F.Gmel.

Basella alba $\mathrm{L}$.

BoerhaviadiffusaL.

Brassica juncea(L.) Czern.

CansjerarheediiJ.F.Gmel.

CanthiumcoromandelicumAlston

CappariszeylanicaL.

Caralluma bicolor Ramach, J. et al., CardiospermumhalicacabumL.

Carissa carandasL.

Carissa spinarumL.

Celosia argentea L.

Cereus pterogonusLem

Cissusquadrangularis L.

Cocciniagrandis(L.) Voigt

CommelinabenghalensisL.

CordiasinensisLam.

CordiadichotomaG.Forst.

CycascircinalisL.

DecalepishamiltoniiWight \&Arn.

Digeramuricata(L.) Mart.

Dioscoreaoppositifolia L.

DioscoreatomentosaJ.König ex Spreng.

DiospyrosmontanaRoxb.

Diospyrosmalabarica(Desr.) Kostel.

DrypetessepiariaWight \&Arn.

ElaeagnusconfertaRoxb.

FicusbenghalensisL.

FicusracemosaL.

Glycosmispentaphylla(Retz.) DC.

GrewiahirsutaVahl

GrewiatiliifoliaVahl

GrewiavillosaWilld.

Hemidesmusindicus(L.)

Ipomoea staphylinaRoem. \&Schult.

JasminumtrichotomumB.Heyneex Roth

Lantana camaraL.

MadhucalongifoliaJ.F.Macbr.

MangiferaindicaL.

MoringaoleiferaLam.

Murrayakoenigii(L.) Spreng.

Opuntiamonacantha(Willd.) Haw.

Opuntiastricta(Haw.) Haw.

Oxalis corniculata L.

Pachygoneovata(Poir.) Diels

Phoenix loureiroiKunth

PhyllanthusemblicaL.

PhyllanthusindofischeriBennet

PhyllanthusreticulatusPoir.

PhysalisangulataL. var. angulata

Pithecellobiumdulce(Roxb.) Benth.

PortulacaoleraceaL.

PsydraxdicoccosGaertn.

RiveahypocrateriformisChoisy

Schleicheraoleosa(Lour.) Merr.

Scutiamyrtina(Burm.f.) Kurz

\begin{tabular}{|c|c|c|}
\hline Liliaceae & Neervekkaea & Tuber \\
\hline Poaceae & Periamungil & Shoot \\
\hline Basellaceae & Vasaladagu & Leaf \\
\hline Nyctaginaceae & Serandai & Leaf \\
\hline Brasscicaceae & Kadugu & Leaf \\
\hline Opiliaceae & Povi & Leaf \\
\hline Rubiaceae & Bellakarai & Fruit \\
\hline Capparaceae & Kevisi & Fruit \\
\hline Asclepiadaceae & Kattalae & Shoot \\
\hline Sapindaceae & Sitiki & Leaf \\
\hline Apocynaceae & Kallakai & Fruit \\
\hline Apocynaceae & Sirukallakai & Fruit \\
\hline Amaranthaceae & Pannae & Leaf \\
\hline Cactaceae & Oocikalli & Flower \\
\hline Vitaceae & Naralai & Leaf \\
\hline Cucurbitaceae & Kovakai & Fruit \\
\hline Commelinaceae & Kannae & Leaf \\
\hline Boraginaceae & Sellai & Leaf \& Fruit \\
\hline Boraginaceae & Karadisellai & Fruit \\
\hline Cycadaceae & Enthu & Tuber \& tender leaf \\
\hline Asclepiadaceae & Magalie & Tuber \\
\hline Amaranthaceae & Theyya & Leaf \\
\hline Dioscoreaceae & Riya & Tuber \\
\hline Dioscoreaceae & Noorai & Tuber \\
\hline Ebenaceae & Bankini & Leaf \\
\hline Ebenaceae & Benson & Fruit \\
\hline Euphorbiaceae & Thanuvam & Fruit \\
\hline Elaeagnaceae & Kolaga & Fruit \\
\hline Moraceae & Aal & Fruit \\
\hline Moraceae & Athi & Fruit \\
\hline Rutaceae & Melaekulukki & Fruit \\
\hline Tiliaceae & Kallai & Fruit \\
\hline Tiliaceae & Lumma & Fruit \\
\hline Tiliaceae & Jenukallai & Fruit \\
\hline Asclepiadaceae & Nannari & Tuber \\
\hline Convolvulaceae & Unnagodi & Tuber \\
\hline Oleaceae & Malligai & Leaf \\
\hline Verbenaceae & Unnichedi & Fruit \\
\hline sapotaceae & Lippae & Fruit \\
\hline Anacardiaceae & Manga & Fruit \\
\hline Moringaeceae & Nugae/Murungai & Leaf \\
\hline Rutaceae & Kariveppilai & Leaf \\
\hline Cactaceae & Kalli & Fruit \\
\hline Cactaceae & Chappathikalli & Fruit \\
\hline Oxalidaceae & Pulichera & Leaf \\
\hline Menispermaceae & Varinkodi & Fruit \\
\hline Arecaceae & Eechipullu & Tender shoot \& fruit \\
\hline Euphorbiaceae & Nelli & Fruit \\
\hline Euphorbiaceae & Nelli & Fruit \\
\hline Euphorbiaceae & Poola & Fruit \\
\hline Solanaceae & Potolai & Fruit \\
\hline Mimosaceae & Konapuli & Fruit \\
\hline Portulacaceae & Goni & Leaf \\
\hline Rubiaceae & Oppai & Fruit \\
\hline Convolvulaceae & Mustae & Leaf \\
\hline Sapindaceae & Pulipoocha & Fruit \\
\hline Rhamnaceae & Sodalie/Julie & Fruit \\
\hline
\end{tabular}


64 Sennatora(L.) Roxb.

65 SolanumamericanumMill.

66 SolanumvirginianumL.

67 SolanumrudepannumDunal

68 StrychnospotatorumL.f.

69 Syzygiumcuminii(L.) Skeels

TamarindusindicaL.

Zaleyadecandra(L.) Burm. f.

ZiziphusmauritianaLam.

Ziziphusoenopolia(L.) Mill.

74 ZiziphusabyssinicaHochst. exA.Rich.

Caesalpiniacea
Solanaceae
Solanaceae
Solanaceae
Loganiaceae
Myrtaceae
Caesalpiniacea
Portulacaceae
Rhamnaceae
Rhamnaceae
Rhamnaceae

Oosithagarai

Leaf

Kakaedagu Leaf

Kandakathiri Fruit

Sundai

Sillakottai

Neera/Naval

Puli

Konidagu

Lanthai

Julie

Kottae

Fruit

Fruit

Fruit

Fruit

Leaf

Fruit

Fruit

Fruit
Table 3. List of families with number of species in st ${ }^{\text {udy }}$ area.

\begin{tabular}{|c|c|c|}
\hline S.NO & Name of the Family & Number of Species \\
\hline 1 & Acanthaceae & 04 \\
\hline 2 & Alangiaceae & 01 \\
\hline 3 & Amaranthaceae & 08 \\
\hline 4 & Anacardiaceae & 01 \\
\hline 5 & Apiaceae & 01 \\
\hline 6 & Apocynaceae & 03 \\
\hline 7 & Araceae & 03 \\
\hline 8 & Arecaceae & 02 \\
\hline 9 & Asclepiadaceae & 04 \\
\hline 10 & Asteraceae & 03 \\
\hline 11 & Basellaceae & 01 \\
\hline 12 & Berberidaceae & 01 \\
\hline 13 & Boraginaceae & 03 \\
\hline 14 & Brasscicaceae & 01 \\
\hline 15 & Cactacea & 03 \\
\hline 16 & Capperaceae & 03 \\
\hline 17 & Caesalpiniaceae & 03 \\
\hline 18 & Chenopodiaceae & 01 \\
\hline 19 & Combretaceae & 02 \\
\hline 20 & Commelinaceae & 01 \\
\hline 21 & Convolvalaceae & 04 \\
\hline 22 & Cucurbitaceae & 03 \\
\hline 23 & Cycadaceae & 01 \\
\hline 24 & Dioscoreaceae & 02 \\
\hline 25 & Ebenaceae & 02 \\
\hline 26 & Elaegnaceae & 02 \\
\hline 27 & Ericaceae & 01 \\
\hline 28 & Erythroxylaceae & 01 \\
\hline 29 & Euphorbiaceae & 08 \\
\hline 30 & Fabaceae & 02 \\
\hline 31 & Lamiaceae & 02 \\
\hline 32 & Liliaceae & 01 \\
\hline 33 & Loganiaceae & 01 \\
\hline 34 & Mackinlayaceae & 01 \\
\hline 35 & Malvaceae & 01 \\
\hline 36 & Meliaceae & 01 \\
\hline 37 & Menispermaceae & 02 \\
\hline 38 & Mimosaceae & 04 \\
\hline 39 & Moraceae & 02 \\
\hline 40 & Moringaceae & 02 \\
\hline
\end{tabular}

\begin{tabular}{|c|c|c|}
\hline 41 & Nyctaginaceae & 01 \\
\hline 42 & Oleaceae & 01 \\
\hline 43 & Opiliaceae & 01 \\
\hline 44 & Oxalidaceae & 01 \\
\hline 40 & Poaceae & 02 \\
\hline 40 & Portulacaceae & 02 \\
\hline 41 & Rhamnaceae & 06 \\
\hline 40 & Rubiaceae & 03 \\
\hline $4 y$ & Rutaceae & 07 \\
\hline ju & Sapindaceae & 04 \\
\hline $\mathrm{J}$ & Sapotaceae & 02 \\
\hline $\mathrm{J}<$ & Solanaceae & 07 \\
\hline כנ & Tiliaceae & 03 \\
\hline 24 & Ulmaceae & 01 \\
\hline כס & Verbinaceae & 03 \\
\hline jo & Vitaceae & 01 \\
\hline ו & Xanthorhoaeaceae & 01 \\
\hline 58 & Zingberaceae & 02 \\
\hline & Total & 140 \\
\hline
\end{tabular}

\section{Fig. 1. Location of the study area}






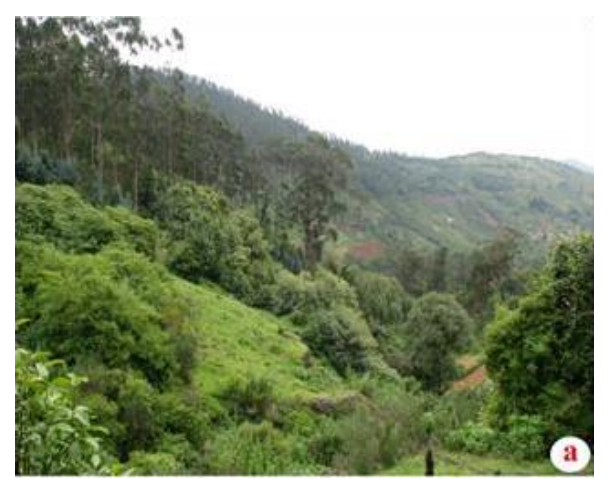

Fig. 2 Overview of the study area.
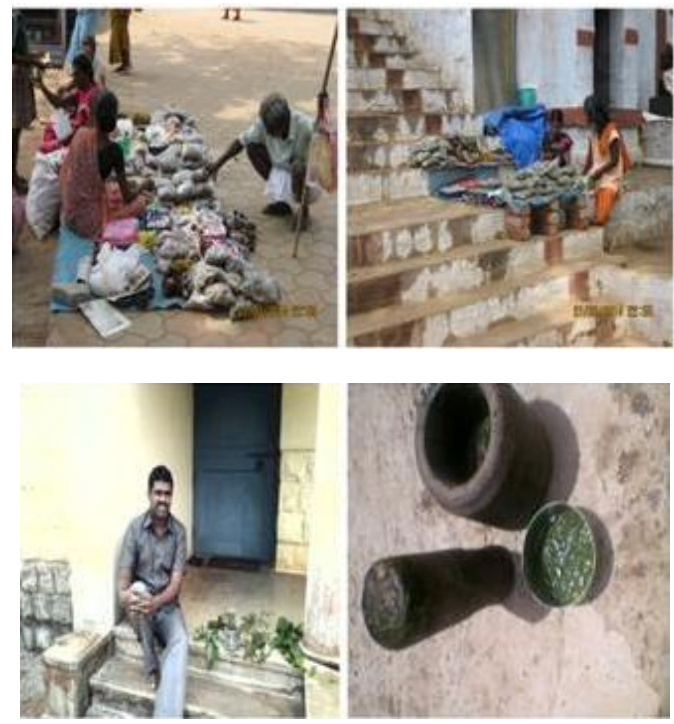

Fig. 3. Preparation of herbal products by Kota tribal in study area
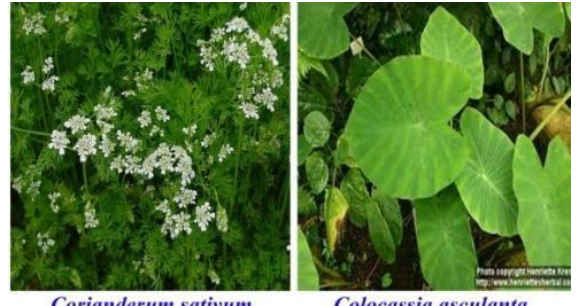

Corianderum sativum Colocassia asculanta

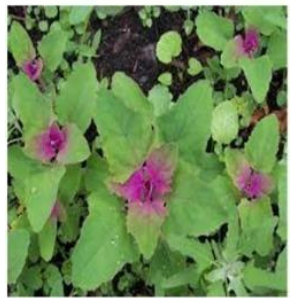

Chenopodium album

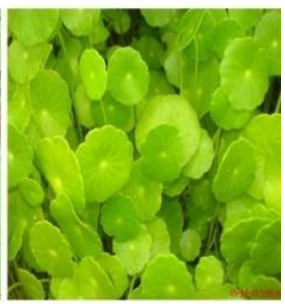

Centella asiatica
Fig. 4. Medicinal plants used by Kota tribal in study area.

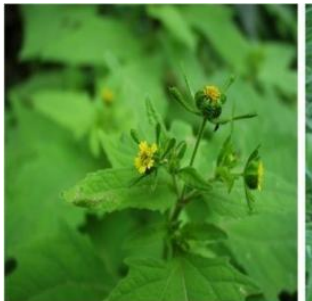

Siegesbeckia orientalis

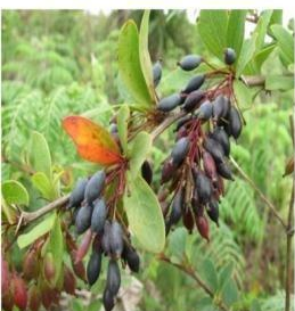

Berberis tinctoria

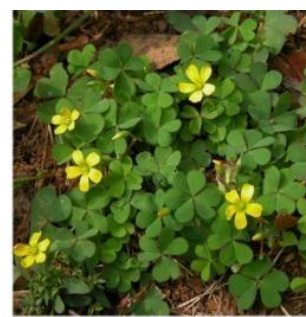

Oxalis corniculata

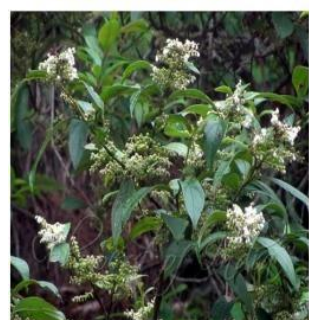

Gaultheria fragrantissima

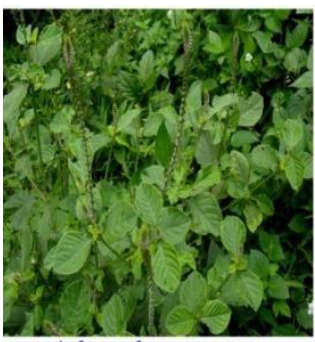

Achranthus aspera

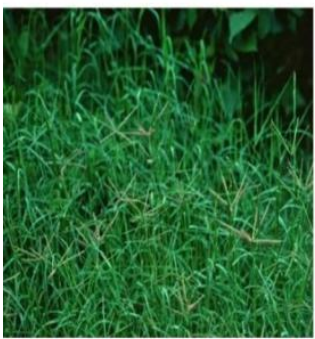

Cynodon dactylon

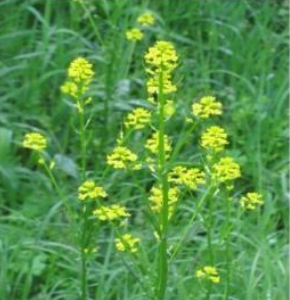

Brassica juncea

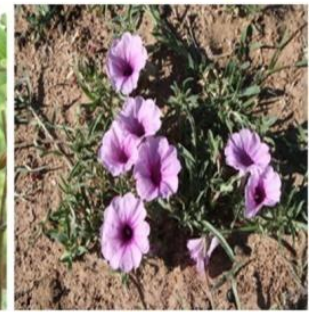

Aroyreia hirsute

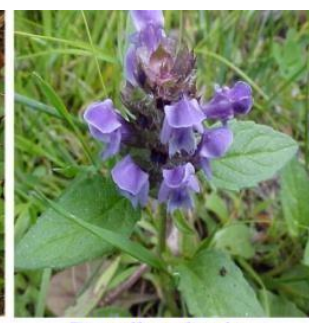

Prunella vulgaris

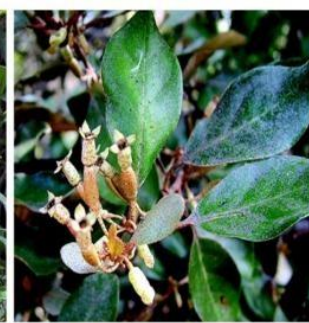

Elaegnus kologa
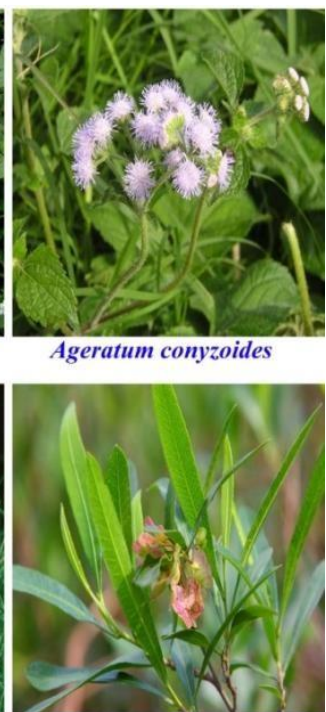

Dodonaea viscosa 
The collected plants are having medicinal properties, which is constructed towards the plant parts like stem, root, leaf and bark etc. Each and every plant differ from mode of utilization as a medicinal and edible food to the human beings and cattle.

\section{CONCLUSION}

In order to study that the traditional uses of these folk medicinal practice day todays life it as resulted providing information of 66 wild plants. Out of the 140 species are comprised in the families like Acanthaceae, Amaranthaceae, Convolvalaceae, Euphorbiaceae, Rutaceae, Solanaceae, etc.,. In this case study, they are used 66 plants in medicinal purpose and 74 plants are used as edible food respectively. So this investigation is held us to understand how indigenous knowledge possess by Kota tribal of study area.

Since most of ethylic communities do not have their own script and return languages the information about the medicinal plants, their dosage, attitude towards disease are unclimbed. So our study is suggested that it is essential to collect information about the use of medicinal plants by the traditional healers and document the same to study them scientifically.

\section{REFERENCES}

Bhattacharjee, S.K., (2001). Hand book of medicinal plants. Pointer publishers, Jaipur, Third revised edition.

Deepak chopra and David Simon, (2000). The Chopra centre herbal hand book. Natural prescription for perfect health and imprint of crown publishing grp, Random House Inc., USA, pp.: 12.

Gamble, J.S., (1936). Flora or the Presidency of Madras. Vol 1-3. Allard and Co. London. (Reprinted- 1956 Botanical Survey of India), Calcutta.

Ganesan, S., N. Suresh and L. Kesavan, (2004). Ethno medicinal survey of lower Palani Hills of Tamilnadu. Indian journal of Traditional Knowledge 3 (3):299 - 304.

Hema, E.S., M. Sivadhasan and N. Anilkumar (2006). Studies on edible species of Amaranthaceae and Araceae used by Kurumba and Pania tribes in Wayanad district, Kerala, India. Ethnobotany 18 : $122-126$.

Kujar, A.A., (1989). A study in cultural geography, (The daughter of St. Anne. Ranchi) pp: 5-7.
Matthew, K.M., (1983). The Flora of Tamilnadu Carnatic. The Rapinact Herbarium, Tiruchirapalli, Tamilnadu.

Murugesan, M., (2005). Floristic diversity and ethnobotanical studies in Velliangiri Hills, the Western Ghats of Coimbatore district, Tamil Nadu, India. Ph.D.Thesis, Bharathiar University, Coimbatore, India.

Paulsamy, S., K.K. Vijaya kumar, M. Murugesan, M. Sivashanmugam,

P. Senthilkumar and D. Suresh, (2008). Medicinal and other economic plants of shola understories, the Nilgiris, Western Ghats. IBD Publisher and Distributors p. 112.

Perumal Samy, R. and S. Ignacimuthu, (2000). Antibacterial activity of some folklore medicinal plants used by tribals in Western Ghats of India. J. Ethno. Pharmaco. 69: 63 - 71.

Poongodi, A., S. Thilagavathi, V. Aravindhan and A. Rajendran, (2011). Observations on some ethno medicinal plants in Sathyamangalam forests of Erode district, Tamil Nadu, India. J. Med. Plant Res. 5:4709-4714.

Prasad, S.M. and P. Balasubramanian, (1996). Strategies for sustainable exploitation of ethnobotanical resources of the Nilgiri Biosphere Reserve, south India. In: S.K. Jain (ed.). Ethnobiol. Human Welfare Deep Publications, New Delhi, pp.331-333.

Rajan, S., M. Sethuraman and P.K. Mukherjee, (2002). Ethnobiology of the Nilgiri Hills, India. Phytother. Res. 16:98-116.

Rajan, S. and M. Sethuraman, (1991). Plants Used In Folk Medicine By The Kotas Of Nilgiri District, Tamil Nadu. Ancient Science of Life. 10(4): 223 230.

Rajan, S., M. Jayendran and M. Sethuraman, (2003). A study on the Kattunayaka tribe of Nilgiri hills, Tamilnadu. J. Nat. Remed. 3(1): 68-72.

Rajasekaran, A., S.N. Prasad and P. Balasubramanian, (2005). Commercial exploited medicinal plants in the Nilgiri Biosphere Reserve, India. J. NonTimber Forest Prod. 12: 8-14.

Rajendran, S.M., S.C. Agarwal and V. Sundaresan, (2004). Lesser Known thnomedicinal Plants of the Ayyakarkoil Forest Province of Southwestern Ghats, Tamil Nadu, India-Part I. J. Herbs, Spices Med. Plants 10: 103 -112.

Rasingam, L. and S.M. Rehel (2009). Major wild edible plants of the Nilgiri Biosphere Reserve in India. Voices 17: 8-9. 
Rasingam, L. (2010). Wild edible Tubers and Rhizomes of the Nilgiri Biosphere Reserve. Newsl. Nilgiri Natur. Hist. Soc. 1: 3.

Revathi, P. and T. Parimelazhagan, (2010). Traditional Knowledge on Medicinal Plants Used by the Irula Tribe of Hasanur Hills, Erode District, Tamil Nadu, India. Ethno botanical Leaflets 14: 136-60.

Udayan, P.S., K.V. Tushar, S. George and I. Balachandran, (2007). Ethnomedicine of the
Chellipale community of Namakal district, Tamil Nadu. Ind. J. Trad. Know. 6: 574 - 578.

Vedavathy, S. (2002). Scope and importance of traditional medicine. Ind. J. Trad. Know. 2(3): 236 - 239.

Yasodharan, K. and K.A. Sujana, (2007). Wild edible plants traditionally used by the tribes in the Parambikulam Wildlife Sanctuary, Kerala, India. Natur. Prod. Rad. 6: 74 - 80. 